\title{
Assembling structures and dynamics properties of perfluorooctane sulfonate (PFOS) at water-titanium oxide interfaces
}

\author{
Guangzhi He, Gang Pan*, Meiyi Zhang \\ Department of Environmental Nano-Materials, Research Center for Eco-Environmental Sciences, Chinese Academy of Sciences, Beijing 100085, China
}

\section{A R T I C L E I N F O}

Article history:

Received 4 March 2013

Accepted 1 May 2013

Available online 14 May 2013

\section{Keywords:}

Perfluorinated surfactant

Interfacial aggregation

Self-assembly

Surface micelle

Counterion bridging

Molecular dynamics simulation

\begin{abstract}
A B S T R A C T
The surface-associated structures and growth modes of perfluorooctane sulfonate (PFOS) at water-rutile $\mathrm{TiO}_{2}$ interfaces were defined by molecular dynamics (MD) simulations. The results showed that a compact PFOS layer was generated at the rutile surfaces, and the assembling structures and dynamic profiles were crystal-face-dependent. PFOS molecules were attached to the (110) and (001) surfaces mainly by the sulfonate headgroups. A well-defined monolayer was formed on the $(110)$ surface with the perfluorinated alkyl chains nearly perpendicular to the substrate, whereas the $\mathrm{C}-\mathrm{F}$ chains were inclined at an angle $\left(30-75^{\circ}\right)$ and formed a hemicylinder-like configuration on the (001) surface. On the other hand, the perfluorinated amphiphiles interacted with the (100) plane through both the sulfonate headgroups (relatively strong electrostatic attraction) and the C-F tailgroups (weak van der Waals forces) and yielded an irregular assembling pattern. Water molecules were mostly concentrated more than $17.0 \AA$ Away from the solid surfaces and formed a continuous solvent layer, suggesting the super hydrophobicity of perfluorinated alkyl chains. A counterion-bridging mechanism suggested in surfactant adsorption was observed at the molecular scale, where the sulfonate headgroups were linked together by the potassium ions at the surfaces and caused the formation of surface aggregates.
\end{abstract}

(c) 2013 Elsevier Inc. All rights reserved.

\section{Introduction}

Perfluorooctane sulfonate (PFOS, $\mathrm{C}_{8} \mathrm{~F}_{17} \mathrm{SO}_{3}^{-}$), as a perfluorinated surfactant, has been manufactured for decades and was used widely in industrial and commercial products [1]. The presence and persistence of PFOS in water and biological samples (fetuses, human milk, and blood) raises public and scientific concerns $[2,3]$. PFOS has been recognized as a global contaminant and was designated as a new member of persistent organic pollutants (POPs) under the Stockholm Convention in 2009 [4]. Different from other POPs, PFOS are highly water-soluble and thus are easy to transport in the aquatic environment [5]. Therefore, the solidwater interfacial process is believed to be a critical factor determining the toxicity and environmental fate of PFOS and has been subjected to intense investigation [6-9].

The hydrophobic perfluoroalkyl chain and hydrophilic sulfonate functional groups are the reasons for the characteristic surfactant behavior of PFOS molecules and provide opportunities to adsorb at a variety of solid-water interfaces. Current studies of PFOS have focused mainly on estimations of global distribution (environment, wildlife, and humans) and sorption isotherm measurements for the

\footnotetext{
* Corresponding author. Fax: +86 1062923541.

E-mail addresses: gzhhe@rcees.ac.cn (G. He), gpan@rcees.ac.cn (G. Pan) myzhang@rcees.ac.cn (M. Zhang).
}

concentration and relative partitioning between the aqueous and solid phases (suspended particles, sediments, and natural minerals) $[6,10-12]$. Electrostatic and hydrophobic interactions have been deduced to play dominant roles in the sorption of PFOS on sediment, clay, and minerals from isotherm experiments $[13,14]$. However, macroscopic experiments provide little information about the surface-associated modes and self-assemble microstructures. In fact, the interaction processes of PFOS with the solidwater interface are complex, changes in the isotherm slope depend on the interactions between hydrophobic tails, repulsions between headgroups and interactions between surfactant molecules with the solid surface $[5,8]$. The mechanisms need to be further verified by atomic-molecular level technologies.

The nature and structure of surfactant aggregates at the solidliquid interface differ from that in bulk solutions due to the solid-surfactant interaction. How aggregation is modified by the presence of solid surfaces is a key issue of surfactant chemistry and surface science [15-17]. The surfaces of natural and synthetic minerals commonly consist of different crystal planes. Different exposed crystal planes on mineral surfaces generally have different geometries, affinity, and catalytic activity to pollutant molecules, and hence may initiate different assembly mechanisms toward perfluoroalkyl compounds (PFCs). Therefore, the crystal planes need to be investigated individually to define the effects of minerals on the interfacial aggregation of PFOS molecules. The solubility 
of PFOS (potassium salt) in pure water is $680 \mathrm{mg} / \mathrm{L}$, which is far below its critical micelle concentration (CMC, $4573 \mathrm{mg} / \mathrm{L}$ ) [18]. Therefore, PFOS molecules fundamentally cannot construct micelle structures in aqueous solution. However, in general, a hemi-micelle state (i.e., surface micelle) would be formed at the solidwater interface when bulk concentration reaches the value of 0.01-0.001 of its CMC (a concentration level of PFOS under experimental and environmental conditions) [14]. Different from the situation in bulk solution, the formation of surface micelle-like aggregates may allow for a compact layer and hence a much higher concentration/density of PFOS at the interface region. However, most of these fundamental properties are extremely difficult to obtain from experiments because some structural transformations (relaxation, disorientation, reorganization, etc.) occur rapidly on the order of nanoseconds.

Molecular dynamics (MD) simulation is able to reproduce the morphological details and structural transitions of complex multiphase systems on the pico-nanosecond (ps-ns) time scales and has been successfully in describing the interfacial processes of organic and biological molecules in the past few years [15,19-21]. Identification of the structure and assembly properties on specific crystal planes from the molecular scale is of fundamental importance to understand the relationship between the crystallographic orientation of powder particles and the adsorption performance toward perfluorinated surfactants. MD simulations have been recently performed to investigate the interfacial aggregation of alkyl surfactants, such as sodium dodecyl sulfate (SDS) and n-alkyl polyethylene oxide [22,23]. The nature of perfluoroalkyl compounds (PFCs) assembled at the solid-water interface may present different features due to the super-hydrophobic and high-strength properties of perfluorinated alkyl chain. However, no reports has been found so far about the interfacial reorganization of perfluorinated surfactants and the transition dynamics from free to micellebound state, which seriously limits the understanding of the fate and behavior of PFCs in the environment.

Here, the equilibrium structures and dynamic properties of PFOS aggregated at the rutile-water interface were studied using the molecular dynamics (MD) simulation. Rutile (the most abundant polymorph of $\mathrm{TiO}_{2}$ ) was chosen as the model substrate because it is one of the most common and technologically important metal oxides for modern science and technology [2427] and showed a much higher affinity to PFOS than Fe and Al oxides in experiments $[5,28]$. An atomistic picture of PFOS adsorption on specific crystal planes was obtained through the MD method. The arrangement and the mobility of sulfonate heads and perfluorinated alkyl tails were quantitatively monitored using the atomic density profiles. Interactions between the perfluorinated surfactants and the role of counterions $\left(\mathrm{K}^{+}\right)$on the surface assembly were
Table 1

Parameters for LJ potential used to model the assembly of PFOS at water/rutile interfaces.

\begin{tabular}{lr}
\hline Atom & Charges $(\mathrm{e})$ \\
\hline $\mathrm{S}$ & 1.474 \\
$\mathrm{O}\left(\right.$ in $\left.\mathrm{SO}_{3}\right)$ & -0.706 \\
$\mathrm{C}\left(\right.$ in $\left.\mathrm{S}_{-}-\mathrm{CF}_{2}\right)$ & 0.166 \\
$\mathrm{C}\left(\right.$ in $\left.\mathrm{CF}_{2}-\mathrm{CF}_{2}\right)$ & 0.520 \\
$\mathrm{C}\left(\right.$ in $\left.\mathrm{CF}_{3}\right)$ & 0.780 \\
$\mathrm{~F}$ & -0.260 \\
$\mathrm{~K}$ & 1.000 \\
$\mathrm{Ti}$ & 1.600 \\
$\mathrm{O}\left(\right.$ in $\left.\mathrm{TiO}_{2}\right)$ & -0.800 \\
$\mathrm{O}\left(\right.$ in $\left.\mathrm{H}_{2} \mathrm{O}\right)$ & -0.820 \\
$\mathrm{H}$ & 0.410 \\
\hline
\end{tabular}

defined with the application of radial distribution functions. On the basis of the detailed information of structures and physical-chemical characteristics, how different crystallographic orientations of the solid modify the perfluorinated surfactant structure on the surface was identified.

\section{Methods}

The simulations of assembling dynamics of PFOS molecules at the water/rutile interfaces were carried out using the Discover module in Materials Studio package (Accerlys Software Inc.). The molecular dynamics simulations were performed with the COMPASS (Condensed-phase Optimized Molecular Potentials for Atomistic Simulation Studies) force field as implemented in the Materials Studio software [29]. COMPASS is an accurate force field for the simulation of molecular interactions in aqueous solutions and has proven to be a reliable description for titanium oxides and surfactant molecules [30-33]. Parameters for Lennard-Jones (LJ) potential used to implement the COMPASS force field are presented in Table 1.

Three crystal planes of rutile ((110), (100), and (001)) were used in the MD simulations. All surfaces were constructed by cleaving the supercells of bulk crystals (see Fig. 1). Dimensions of the solid walls and number of $\mathrm{TiO}_{2}$ molecules used to build the solid surfaces are presented in Table 2 . The initial configuration of the interfacial system was prepared from a monolayer of 36 PFOS molecules (a length of $\sim 1.0 \mathrm{~nm}$, the structure is shown in Fig. 1a and Table 3 ) with the sulfonate headgroups pointed to the surface ( $\sim 8 \AA$ above the substrate), which yielded a surface coverage of $\sim 2.0$ molecules $/ \mathrm{nm}^{2}$ on the three rutile planes. A similar surface density level was also employed to investigate the interfacial assembly of long-chain-length alkyl sulfate surfactants [15,34].

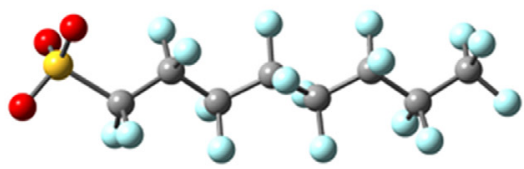

a

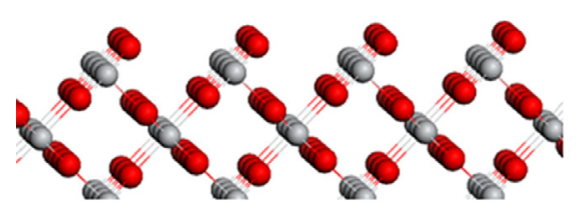

C

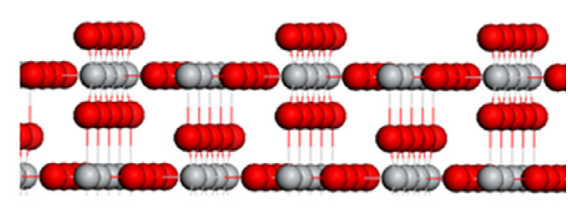

b

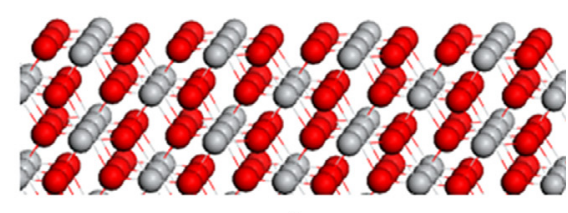

d

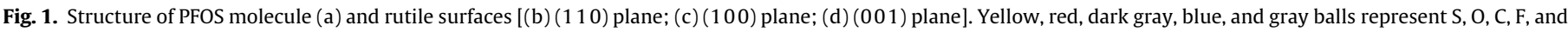
$\mathrm{Ti}$ atoms, respectively. (For interpretation of the references to color in this figure legend, the reader is referred to the web version of this article.) 
Table 2

Spatial dimensions and number of $\mathrm{TiO}_{2}$ molecules used to build the solid surfaces.

\begin{tabular}{lllll}
\hline Plane & $L_{x}(\AA)$ & $L_{y}(\AA)$ & $L_{z}(\AA)$ & $n_{\mathrm{TiO} 2}$ \\
\hline$(110)$ & 41.42 & 38.98 & 12.99 & 766 \\
$(001)$ & 41.35 & 41.35 & 10.36 & 842 \\
$(100)$ & 41.36 & 41.43 & 13.78 & 840 \\
\hline
\end{tabular}

Table 3

Structural parameters of PFOS molecule.

\begin{tabular}{llll}
\hline Distance & Value $(\AA)$ & Angle & Value $\left(^{\circ}\right)$ \\
\hline S-O & 1.48 & S-C-C & 116 \\
S-C & 1.91 & C-C-C & 114 \\
C-C & 1.57 & & \\
C-F & 1.36 & & \\
\hline
\end{tabular}

Then $\mathrm{K}^{+}$ions were added close to the headgroups to neutralize the charge of anionic PFOS (i.e., the potassium salt of PFOS). To reach a water density of $\sim 1.0 \mathrm{~g} / \mathrm{cc}$ within the solution layer of $40 \times 40 \times 40 \AA^{3}$, 2000 water molecules were used to simulate the solvent environment. The water was simulated using the simple point charge (SPC) model [35], which worked reliably with the COMPASS force field. The crystal atoms were constrained to their equilibrium bulk positions during the simulations [31,36]. Periodic boundary conditions were used, and the slabs were separated from their periodic images normal to the surface by a vacuum gap of $60 \AA$ to prevent the interactions between the periodic replicas in the $Z$ direction $[15,37]$. The resulted final box was approximately $40 \times 40 \times 120 \AA^{3}$. The MD simulation was carried out without the consideration of surface hydroxyl groups at present, due to the difficulty in describing the behavior of $-\mathrm{H}_{2} \mathrm{O} /-\mathrm{OH}$ surface groups using current force fields [34,37]. With the development of molecular dynamics techniques, it would be possible to simulate a wide range of water/hydroxyl coordination structures and produce a better description of interface-related processes in the future.

The simulations were initialized by a 5000-step energy minimization of the initial configurations using the smart minimizer method, which switches from steepest-descent to conjugated gradient method as the energy derivatives decrease in order to accelerate the computation. After the initialization, all the MD simulations were carried out under the canonical ensemble (NVT) with a time step of $1 \mathrm{fs}$. The equations of motion were integrated with the velocity Verlet algorithm [38]. Initial velocities were randomly assigned according to Boltzmann distribution. The Andersen thermostat with collision ratio of 1.0 was used to maintain a constant system temperature of $298 \mathrm{~K}$ [39]. The electrostatic interactions were handled with the Ewald method [40], and the Van der Waals interactions were calculated by atom-based summation method using a cutoff distance of $9.5 \AA$ with longrange corrections added. Finally, all the systems were equilibrated for $20 \mathrm{~ns}$, and the data from the last $5 \mathrm{~ns}$ were collected for analysis. The simulation was confirmed to have reached equilibrium within 20 ns by monitoring the temperature, potential energy, and the density profiles of PFOS molecules $\left(-\mathrm{CF}_{3}\right.$ tailgroups) as a function of time (see the Supporting information).

\section{Results and discussion}

\subsection{Rutile $\mathrm{TiO}_{2}$ surfaces}

The (110) and (100) facets contribute to about $60 \%$ and $20 \%$ of the total crystal surface of natural rutile powder, respectively [41], and thus, they constitute the dominant surfaces for adsorption reaction. The lowest-energy (110) surface consists of a basal plane of $\mathrm{O}$ and $\mathrm{Ti}$ atoms with overlying rows of the bridging oxygen atoms, and the bridging oxygens are oriented in a plane perpendicular to the basal surface (Fig. 1b). The (100) face shows a sawtooth pattern created by bridging $\mathrm{O}$ atoms connected to the five-coordinated $\mathrm{Ti}$ atoms, where the bridging plane $\mathrm{Ti}-\mathrm{O}-\mathrm{Ti}$ is inclined at an angle (Fig. 1c). Rutile (001) face exhibits $-\mathrm{O}-\mathrm{Ti}-\mathrm{O}-\mathrm{Ti}-$ rows with two kinds of atoms: four-coordinated $\mathrm{Ti}$ atoms and twofold $\mathrm{O}$ atoms (Fig. 1d). Because of the large-coordinate unsaturation of Ti atoms, the (001) face displays a higher surface reactivity than other rutile faces [41]. Therefore, the three types of rutile surfaces were employed to determine the interfacial structures and dynamic profiles of PFOS molecules.

\subsection{Equilibrium structures}

In order to quantify the interaction between the perfluorinated surfactants and the substrate, the density profiles along the $Z$ direction (i.e., normal to the solid surfaces) for the sulfonate headgroups and the $-\mathrm{CF}_{3}$ tailgroups were calculated (Fig. 2). The snapshots of the final structures of PFOS aggregated on the three different crystal planes of rutile are present in Fig. 3. The results showed that the PFOS molecules interacted with the (110) and (001) surfaces mainly by their sulfonate headgroups at the distance of 2.5-3.2 $\AA$ (see the peaks of the $-\mathrm{SO}_{3}$ groups in Fig. 2a and $b$ ), but the shape and orientation of the aggregates were different. The density profiles on the (110) plane showed a much sharper peak of the tailgroups (at $\sim 13.2 \AA$ ), suggesting that the PFOS were better ordered than those on the (001) plane. Specifically, PFOS molecules arrayed in a well-defined monolayer on the (110) surface with a direction nearly normal to the substrate, whereas the perfluoroalkyl chains were inclined to the (001) surface at an angle $\left(30-75^{\circ}\right)$ and formed a hemicylinder micelle-like structure (Fig. 3a and b). On the (100) surface, PFOS displayed a clearly different assembling behavior to that on both (110) and (001) surfaces in terms of the morphology and layer formation (Fig. 3c). The perfluorinated amphiphilic molecules were not all attached to the (100) surface by the hydrophilic sulfonate headgroups. More than $1 / 3$ of the PFOS molecules interacted with the (100) substrate by their hydrophobic C-F chains (see the peak of the $-\mathrm{CF}_{3}$ groups at $\sim 3.6 \AA$ in Fig. $2 \mathrm{c}$ ), showing a weak hydrophobicity of rutile (100) facet. The shape of the equilibrium configuration on the (100) surface was not as regular as that on the (110) and (001) surfaces, partially forming a second layer (see the weak peak of the $-\mathrm{SO}_{3}$ groups at $\sim 18.5 \AA$ in Fig. $2 \mathrm{c}$ ).

It has been known that the interfacial morphology of surfactants depends on the chain length. In general, the surfactants with short alkyl chains easily form monolayers, longer-tail surfactants are able to generate semicylinder configurations, and the aggregates usually show irregular multilayer shapes under high surfactant concentration conditions [22,42]. The three typical surface assembling structures were, respectively, observed in the aggregation of medium-chain-length PFOS molecules (eight perfluorinated carbon atoms) at the three different surfaces of rutile. Changes in surfactant aggregate structures can cause pronounced changes of local chemical properties and surface reactivity (such as binding strength). The MD results suggested a possible way of dispersing the powder particles exposed different crystal planes in water to trap and manipulate the reversibility and bioavailability of perfluoroalkyl compounds (PFCs) through altering the interaction strength and the components of force (i.e., electrostatic attraction and van der Waals forces).

The $(100)$ face exhibits the same three kinds of atoms as the (110) face (five-coordinated $\mathrm{Ti}$ atoms, two-coordinated $\mathrm{O}$ atoms and three-coordinated $\mathrm{O}$ atoms), but the bridging plane $\mathrm{Ti}-\mathrm{O}-\mathrm{Ti}$ is inclined at an angle, rather than normal to the basal plane as 


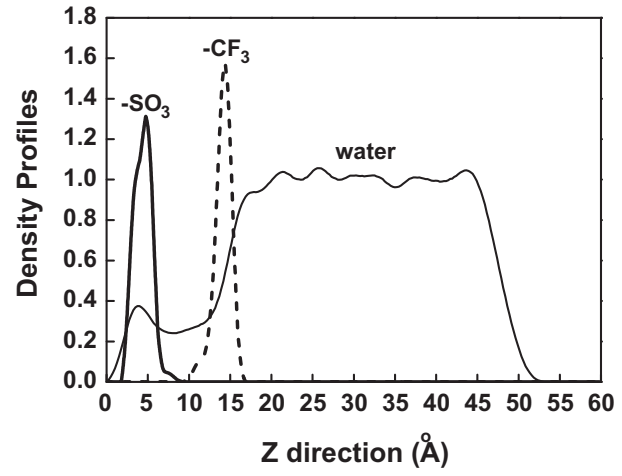

a

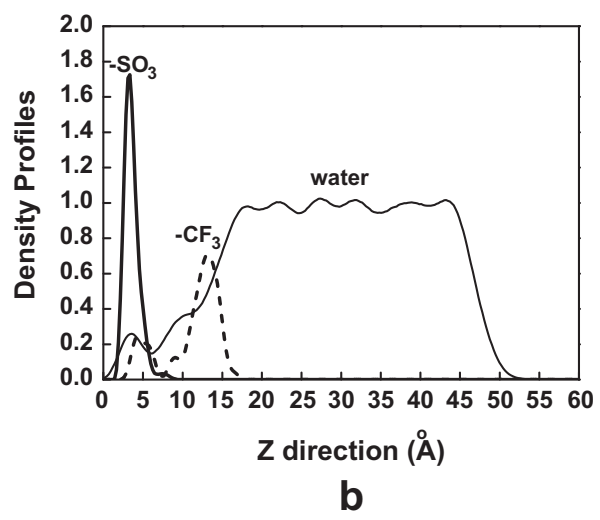

b

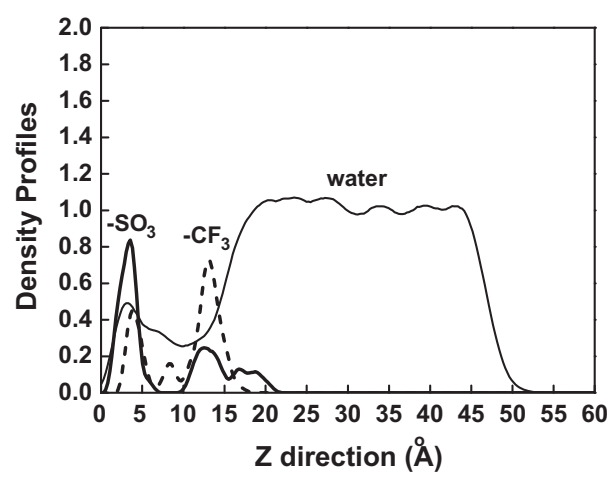

C

Fig. 2. Density profiles of PFOS and water molecules along the $Z$ direction at different surfaces of rutile: (a) (110) plane; (b) (001) plane; (c) (100) plane.

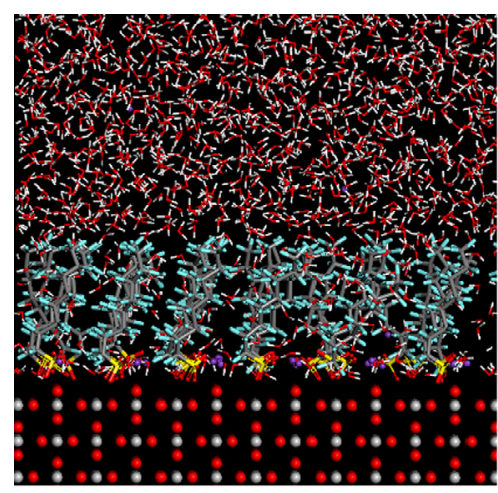

a

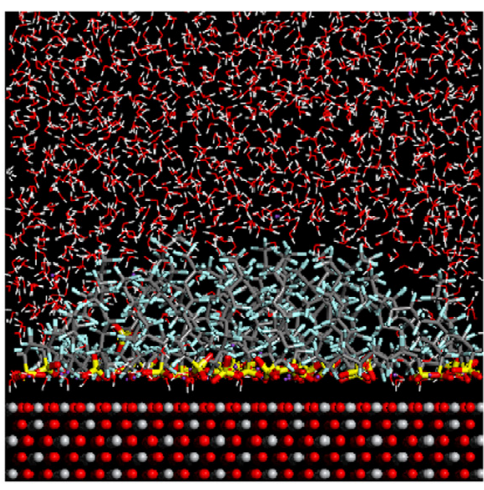

b

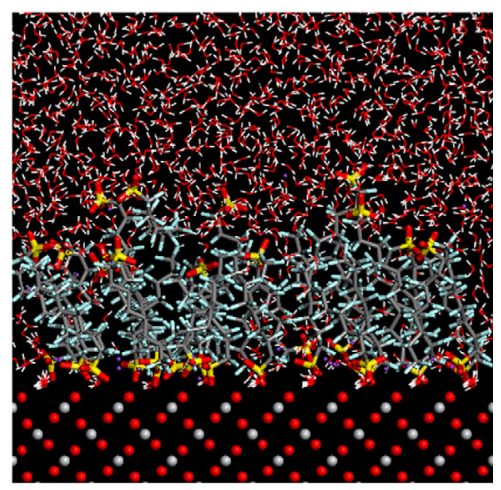

C

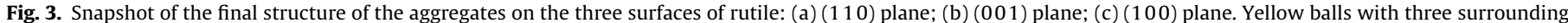

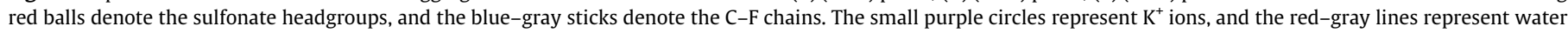

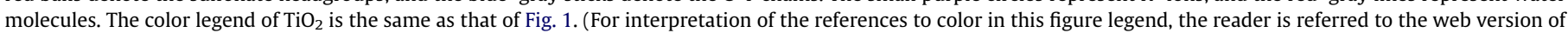
this article.)

in (110) face (see Fig. 1b and c). Our MD results indicated that the atomic orientation of solid surfaces directly affected its hydrophilicity and imposed a difference toward the surfactant-solid interactions. As shown in Fig. 3, the formation of monolayer structure on (110) substrate was caused primarily by interactions between the sulfonate headgroups with the solid surfaces through electrostatic attraction. However, the perfluorinated amphiphiles interacted with the (100) plane through both the sulfonate groups (electrostatic attraction) and the perfluoroalkyl chains (van der Waals forces) and yielded a relatively irregular assembling pattern. This result suggested that the $(100)$ surface was not as hydrophilic as the (110) and the (001) surfaces, a finding consistent with the experimental measurement of hydrophobicity using contact angles of water drops [43]. The hydrophilic (110) facet is the dominant exposed crystal plane of rutile particles, while the weak hydrophobic (100) facet has a minor contribution to the powder surfaces. Therefore, rutile displays hydrophilic properties in aqueous solution under ambient conditions.

\subsection{Interaction between water and PFOS molecules}

The density profiles showed that water molecules were mainly concentrated more than $17.0 \AA$ away from the solid surface and formed a continuous solvent layer (Fig. 2). However, it was worth 


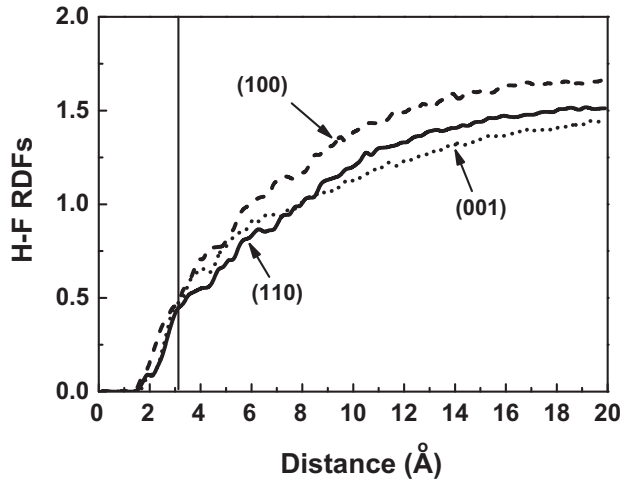

a

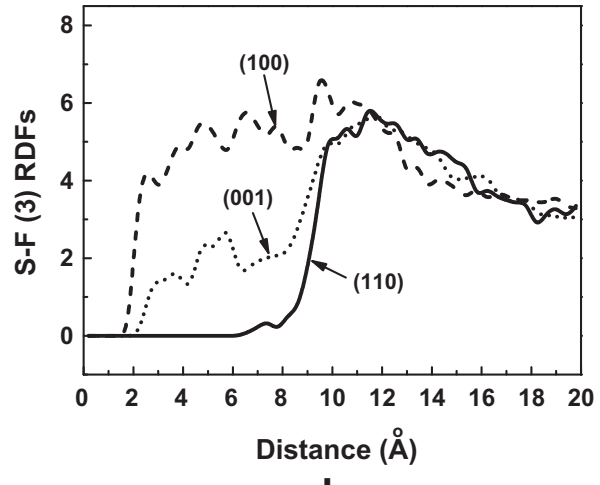

b

Fig. 4. (a) H-F and (b) S-F(3) radial distribution functions on the three crystal planes.
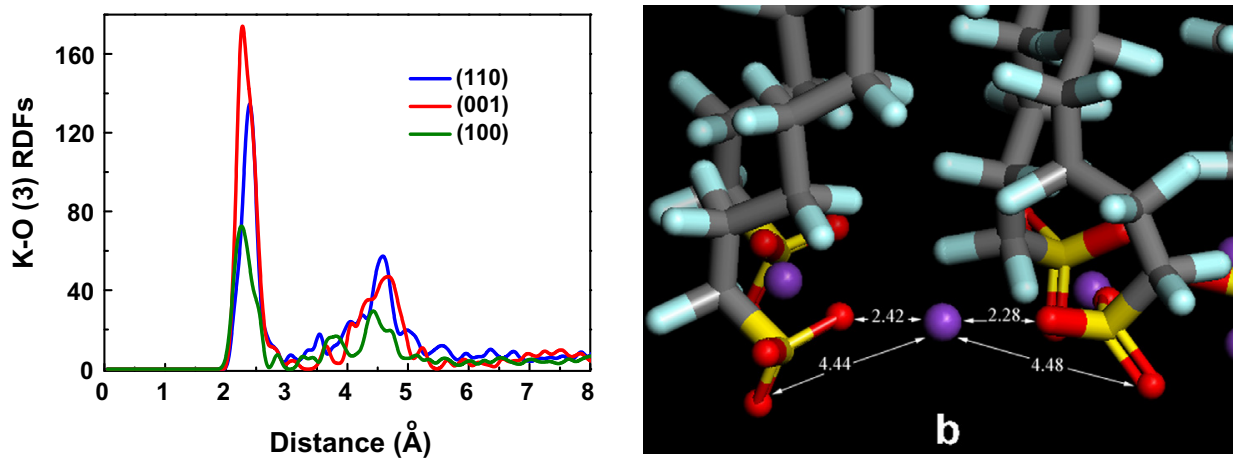

a

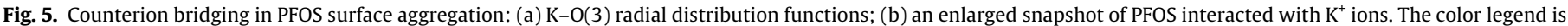
the same as that of Fig. 3, and distances are shown in angstroms.

to note that there was a small peak of water at the distance of 2.0$4.5 \AA$ above the solid. Here, the water molecules were hydrogenbonded to the sulfonate headgroups (typically $\sim 1.5 \AA$ ) and oxygen atoms of solid surfaces (typically $\sim 1.9 \AA$ ), generating a thin solvent layer near the surfaces.

To characterize the interaction between the perfluorinated alkyl chains and water molecules, the $\mathrm{H}-\mathrm{F}$ radial distribution functions (RDFs) were calculated. This result showed that water molecules mainly located more than $3.1 \AA$ away from the $\mathrm{C}-\mathrm{F}$ chains (Fig. 4a), which was longer than the $\mathrm{O} \cdots \mathrm{H}(\mathrm{C})$ hydrogen bond lengths between the $\mathrm{C}-\mathrm{H}$ chains and water (typically $2.2-2.6 \AA$ ) [44], suggesting the super-hydrophobic property of perfluorinated alkyl chains.

The self-interaction features of PFOS molecules were defined by the $\mathrm{S}-\mathrm{F}(3)$ radial distribution functions $(\mathrm{F}(3)$, the $\mathrm{F}$ atoms on the $\mathrm{CF}_{3}$ tailgroups). The $\mathrm{S}-\mathrm{F}(3) \mathrm{RDF}$ showed that the $-\mathrm{CF}_{3}$ groups were primarily located $\sim 10 \AA$ away from the sulfonate groups (see the main peaks in Fig. $4 \mathrm{~b}$ ), a value corresponding to the length of PFOS molecule. A broad peak was observed at the distance of 3.0-7.0 in the case of (001) and (100) planes, suggesting a weak interaction between the perfluorinated surfactants in the hemicylinder and irregular aggregates. The broad peak was more pronounced for the irregular organization on $(100)$ plane, but almost no $-\mathrm{CF}_{3}$ tailgroups were found within the distance of $\sim 5.0 \AA$ from a sulfur atom in the well-ordered monolayer on (110) plane (Fig. $4 \mathrm{~b}$ ). This result indicated that the first peak of S-F(3) RDFs can be used to qualitatively estimate the disorder of surfactant molecules assembled on solid surfaces.

\subsection{Counterion-bridging effects}

To investigate the role of the counterions (i.e., $\mathrm{K}^{+}$) in the interfacial aggregation of PFOS molecules, the $\mathrm{K}-\mathrm{O}(3) \mathrm{RDFs}(\mathrm{O}(3)$, the $\mathrm{O}$ atoms on the $-\mathrm{SO}_{3}$ headgroups) were analyzed (Fig. 5a). An enlarged snapshot of PFOS interacted with $\mathrm{K}^{+}$is present in Fig. $5 \mathrm{~b}$. This radial distribution function analysis allowed us to quantify the counterion-bridging phenomenon in PFOS adsorption. In the equilibrium configurations, potassium ions associated with the sulfonate headgroups at the surfaces, neutralizing the chargecharge repulsion between the negative sulfonate groups and instead inducing an effective attraction. The $\mathrm{K}-\mathrm{O}(3)$ RDFs were dominated by two peaks at the positions of $\sim 2.4$ and $\sim 4.5 \AA$ (Fig. 5a), which corresponded to the distances between the $\mathrm{K}^{+}$and the neighbor and non-neighbor $\mathrm{O}$ atoms of two adjacent sulfonate groups (Fig. 5b). This attraction was strong enough to link the surfactant molecules and cause formation of the surface aggregates shown in Fig. 3.

\section{Conclusions}

The molecular dynamics (MD) results indicated that the structure and crystallographic orientation of solid surfaces directly affected the surfactant-solid interactions (structures and the components of force). PFOS molecules were attached to the (110) and (001) surfaces mainly by the sulfonate headgroups. A well-defined monolayer was formed on the (110) facet, whereas 
the perfluorinated alkyl chains were inclined at an angle and formed a hemicylinder-like configuration on the (001) facet. The perfluorinated amphiphiles were not all attached to the (100) surface by the hydrophilic sulfonate headgroups. More than $1 / 3$ of the PFOS molecules interacted with the (100) substrate by their hydrophobic C-F chains and generated an irregular assembling pattern. The super hydrophobicity of perfluoroalkyl chains and the counterion-bridging phenomenon were quantitatively determined by radial distribution function analysis. By offering detailed structural and dynamic information, MD simulation technique may provide a new perspective on perfluorinated surfactants from atomic/molecular scale.

\section{Acknowledgments}

The study was supported by NNSF of China (21207151, 21277161, 21007083, and 41103076), the special fund of State Key Joint Laboratory of Environment Simulation and Pollution Control (12L02ESPC), and the special fund from the State Key Laboratory of Environmental Aquatic Chemistry (11Y06ESPCR). We thank the Supercomputing Center of the Chinese Academy of Sciences for providing access to the Materials Studio software.

\section{Appendix A. Supplementary material}

Supplementary data associated with this article can be found, in the online version, at http://dx.doi.org/10.1016/j.jcis.2013.05.003.

\section{References}

[1] K. Prevedouros, I.T. Cousins, R.C. Buck, S.H. Korzeniowski, Environ. Sci. Technol. 40 (1) (2006) 32-44.

[2] K. Kannan, S. Corsolini, J. Falandysz, G. Fillmann, K.S. Kumar, B.G. Loganathan, M.A. Mohd, J. Olivero, N. Van Wouwe, J.H. Yang, K.M. Aldous, Environ. Sci. Technol. 38 (17) (2004) 4489-4495.

[3] C.E. Mueller, A.O. De Silva, J. Small, M. Williamson, X. Wang, A. Morris, S. Katz M. Gamberg, D.C.G. Muir, Environ. Sci. Technol. 45 (20) (2011) 8665-8673.

[4] T. Wang, Y. Wang, C. Liao, Y. Cai, G. Jiang, Environ. Sci. Technol. 43 (14) (2009) 5171-5175.

[5] F. Wang, K. Shih, Water Res. 45 (9) (2011) 2925-2930.

[6] C. Lau, K. Anitole, C. Hodes, D. Lai, A. Pfahles-Hutchens, J. Seed, Toxicol. Sci. 99 2) (2007) 366-394

[7] G. Pan, C. You, Environ. Pollut. 158 (5) (2010) 1363-1367.

[8] Q. Yu, R. Zhang, S. Deng, J. Huang, G. Yu, Water Res. 43 (4) (2009) 1150-1158.
[9] Q. Zhou, S. Deng, Q. Zhang, Q. Fan, J. Huang, G. Yu, Chemosphere 81 (4) (2010) 453-458.

[10] J.W. Martin, D.M. Whittle, D.C.G. Muir, S.A. Mabury, Environ. Sci. Technol. 38 (20) (2004) 5379-5385.

[11] C. You, C. Jia, G. Pan, Environ. Pollut. 158 (5) (2010) 1343-1347.

[12] F. Xiao, K.J. Davidsavor, S. Park, M. Nakayama, B.R. Phillips, J. Colloid Interface Sci. 368 (2012) 505-511.

[13] C.P. Higgins, R.G. Luthy, Environ. Sci. Technol. 40 (23) (2006) 7251-7256.

[14] R.L. Johnson, A.J. Anschutz, J.M. Smolen, M.F. Simcik, R.L. Penn, J. Chem. Eng. Data 52 (4) (2007) 1165-1170.

[15] H. Dominguez, Langmuir 25 (16) (2009) 9006-9011.

[16] S. Paria, K.C. Khilar, Adv. Colloid Interface Sci. 110 (3) (2004) 75-95.

[17] C. de Gracia Lux, J.-L. Gallani, G. Waton, M.P. Krafft, Chem. Eur. J. 16 (24) (2010) 7186-7198.

[18] E. Kissa, Fluorinated Surfactants and Repellents, second ed., Marcel Dekker New York, 2001.

[19] J. O’Young, Y. Liao, Y. Xiao, J. Jalkanen, G. Lajoie, M. Karttunen, H.A. Goldberg, G.K. Hunter, J. Am. Chem. Soc. 133 (45) (2011) 18406-18412.

[20] A. Kornherr, G.E. Nauer, A.A. Sokol, S.A. French, C.R.A. Catlow, G. Zifferer, Langmuir 22 (19) (2006) 8036-8042.

[21] E. Ryjkina, H. Kuhn, H. Rehage, F. Muller, J. Peggau, Angew. Chem., Int. Ed. 41 (6) (2002) 983-986

[22] G. Srinivas, S.O. Nielsen, P.B. Moore, M.L. Klein, J. Am. Chem. Soc. 128 (3) (2006) 848-853.

[23] N.R. Tummala, A. Striolo, J. Phys. Chem. B 112 (7) (2008) 1987-2000.

[24] U. Diebold, Surf. Sci. Rep. 48 (5-8) (2003) 53-229.

[25] G. He, G. Pan, M. Zhang, J. Synchrotron. Radiat. 19 (2012) 394-399.

[26] G.Z. He, M.Y. Zhang, G. Pan, J. Phys. Chem. C 113 (52) (2009) 21679-21686

[27] A. Fujishima, X. Zhang, D.A. Tryk, Surf. Sci. Rep. 63 (12) (2008) 515-582.

[28] Q.Z. Yuan, R. Ravikrishna, K.T. Valsaraj, Sep. Purif. Technol. 24 (1-2) (2001) 309-318.

[29] H. Sun, J. Phys. Chem. B 102 (38) (1998) 7338-7364.

[30] X. Zhang, G. Lu, X. Wen, H. Yang, Appl. Surf. Sci. 255 (13-14) (2009) 64936498.

[31] B. Prathab, V. Subramanian, T.M. Aminabhavi, Polymer 48 (1) (2007) 409-416

32] A. Kornherr, A. Tortschanoff, E. Portuondo-Campa, F. van Mourik, M. Chergui, G. Zifferer, Chem. Phys. Lett. 430 (4-6) (2006) 375-379.

[33] A. Kornherr, D. Vogtenhuber, M. Ruckenbauer, R. Podloucky, G. Zifferer, J. Chem. Phys. 121 (8) (2004) 3722-3726.

[34] E. Nunez-Rojas, H. Dominguez, J. Colloid Interface Sci. 364 (2) (2011) 417-427.

[35] H.J.C. Berendsen, J.R. Grigera, T.P. Straatsma, J. Phys. Chem. 91 (24) (1987) 6269-6271.

[36] C. Li, P. Choi, J. Phys. Chem. C 111 (4) (2007) 1747-1753.

[37] S. Monti, A.C.T. van Duin, S.-Y. Kim, V. Barone, J. Phys. Chem. C 116 (8) (2012) 5141-5150

[38] L. Verlet, Phys. Rev. 159 (1) (1967) 98

[39] H.C. Andersen, J. Chem. Phys. 72 (4) (1980) 2384-2393.

[40] S. Plimpton, J. Comput. Phys. 117 (1) (1995) 1-19.

[41] R.S. Kavathekar, P. Dev, N.J. English, J.M.D. MacElroy, Mol. Phys. 109 (13) (2011) 1649-1656.

[42] H. Dominguez, J. Phys. Chem. B 115 (43) (2011) 12422-12428.

[43] R. Wang, N. Sakai, A. Fujishima, T. Watanabe, K. Hashimoto, J. Phys. Chem. B 103 (12) (1999) 2188-2194.

[44] T. Kar, S. Scheiner, J. Phys. Chem. A 108 (42) (2004) 9161-9168. 